\title{
Evaluation of advanced polymers for additive manufacturing ${ }^{1}$ PPG Industries, Inc.
}

\author{
Project ID: $\quad$ MDF-TC-2014-048 \\ Start Date: $\quad$ 7/8/2014 \\ Completion Date: 12/31/2014 \\ Company Size: Large business
}

\section{Summary}

The goal of this Manufacturing Demonstration Facility (MDF) technical collaboration project between Oak Ridge National Laboratory (ORNL) and PPG Industries, Inc. was to evaluate the feasibility of using conventional coatings chemistry and technology to build up material layer-by-layer. The PPG-ORNL study successfully demonstrated that polymeric coatings formulations may overcome many limitations of common thermoplastics used in additive manufacturing (AM), allow lightweight nozzle design for material deposition and increase build rate. The materials effort focused on layer-bylayer deposition of coatings with each layer fusing together. The combination of materials and deposition results in an additively manufactured build that has sufficient mechanical properties to bear the load of additional layers, yet is capable of bonding across the $z$-layers to improve build direction strength. The formulation properties were tuned to enable a novel, high-throughput deposition method that is highly scalable, compatible with high loading of reinforcing fillers, and is inherently low-cost.

\section{Background}

Thermoplastics such as Nylon, ABS, and PEEK are the status-quo in polymer additive manufacturing. However there are several limitations associated with thermal based deposition systems including complex methods of thermal control, part distortion, and weak layer to layer strength. In traditional polymer extrusion-based systems the feed material is simply melted and extruded directly onto a cold or warm plate or prior build layer. Although simple in design, this method requires materials that are spatially locked in place immediately after deposition, maintain tolerance during subsequent thermal

\footnotetext{
${ }^{1}$ Research sponsored by the U.S. Department of Energy, Office of Energy Efficiency and Renewable Energy, Advanced Manufacturing Office, under contract DE-AC05-00OR22725 with UT-Battelle, LLC.
} 
cycling and form a strong a mechanical bond to subsequent layers. The mechanical strength of a thermoplastic typically increases with the molecular weight and degree of branching or side chains. Unfortunately this also results in an elevation of the melt viscosity and melting point. The z-strength or mechanical properties of the bond between adjacent layers is formed by physically pushing the polymer melt into the previous layer. Therefore the resistance to melt flow is an important parameter, and the extrusion of high strength thermoplastics requires elevated temperatures that aggravate thermal distortion. Alternative materials for AM need to outperform thermoplastics in a number of critical areas, including mechanical properties (such as elastic modulus), chemical resistance, thermal stability, and overall durability. New materials that can be used in composite structures, and can attain higher performance than thermoplastic composites, are particularly desired.

\section{Technical Results}

Polymeric coating formulations for direct print additive manufacturing were determined to be feasible, and in some aspects superior to traditional additive manufacturing. The properties are tunable via slight modifications in the formulations. PPG provided 16 different coating formulations and ORNL characterized the behavior, deposition and mechanical properties of cast neat and carbon fiber (CF) reinforced additively manufactured parts.

ORNL also employed the recently developed drop flow test. This simple test consists of depositing the coating formulation at a constant rate onto a flat room temperature or heated surface for a predefined time interval. The height, width and morphology of the "drop" are used to characterize the material's ability to form free standing structures.

In order to demonstrate the viability of coatings formulations in additive manufacturing, a bead forming experiment was also performed. In this experiment peristaltic pumps were used in order to control the flow rate of the formulation. The mixed polymer was extruded onto a flat surface, simulating what would happen in an additive manufacturing system.

It was determined from the tests described above that the coatings formulations were not viscous enough to form a bead in pure form, and did not pump evenly due to the differing viscosities of the various components. Additives were used to increase the viscosity of the individual components to a gel like consistency prior to pumping and mixing. The additives impart shear thinning and yield stress behavior to the formulation by forming a weak network in suspension. At very low stress, the weak network provides a finite elastic stiffness that prevents sagging and viscous flow. Under applied shear stress (e.g. extrusion through a nozzle), the weak network breaks up and the material is able to flow freely at low viscosity. Once the material is extruded out of the nozzle, the shear stress is relaxed and the weak network reforms, enabling the printed material to maintain its shape. 


\section{Impacts}

Promising results from this Phase $1 \mathrm{MDF}$ technical collaboration project have given PPG renewed confidence in leveraging their expertise in polymeric coatings formulations to increase the materials that are available for additive manufacturing applications. While the current study was limited, we believe that a much larger spectrum of coatings formulations will also be accessible. Continued work will facilitate optimization of the coatings formulations studied in this phase, but will also direct the development of new systems with a much broader range of ultimate properties which can address end-use applications currently inaccessible using traditional thermoplastics. PPG is a materials company specializing in polymeric materials synthesis, characterization, and development for a broad range of end-use applications including organic coatings, optical materials, and adhesives. Unlike many other coatings companies, PPG has expertise in polymer design and manufactures most of its own bulk resins and coatings binder materials. Through partnering with the MDF, we hope to develop new AM materials and associated equipment. By including polymeric coatings formulations in the toolbox for AM end users, a new category of parts with high performance will be available. The ultimate impact will be to expand the AM design space and to provide a new market for manufacturers of thermoset material.

\section{Conclusions}

Polymeric coatings formulations for direct print additive manufacturing were determined to be feasible and in some aspects superior to traditional additive manufacturing. Direct deposition methods partially developed under this technical collaboration were shown to lighten the nozzle and overcome this inherent limitation in throughput. A gantry style 3D printer that is comparable in design to Big Area Additive Manufacturing (BAAM) was modified for direct deposition of polymer coatings formulations to form free standing structures. The build rate was increased dramatically and a joint patent was filed that includes inventors from PPG and ORNL.

\section{About the Company}

PPG is currently the world's leading maker of transportation coatings and a major global supplier of industrial and packaging coatings, employing over 38,000 people in more than 150 facilities worldwide. Over 40 of those facilities and roughly 14,000 employees are located in the United States. PPG Industries' vision is to continue to be the world's leading coatings and specialty products company. Through leadership in innovation, sustainability and color, PPG helps customers in industrial, transportation, consumer products, and construction markets and aftermarkets to enhance more surfaces in more ways than does any other company.

\section{Points of Contact:}

Orlando Rios, rioso@ornl.gov

Crystal Morrison, cmorrison@ppg.com 